\title{
Retz et Mazarin: polémique et politique dans les "Mémoires" de Retz
}

\section{Bruno Tribout}

\section{(2) OpenEdition}

1 Journals

\section{Édition électronique}

URL : https://journals.openedition.org/studifrancesi/2941

DOI : 10.4000/studifrancesi.2941

ISSN : 2421-5856

Éditeur

Rosenberg \& Sellier

\section{Édition imprimée}

Date de publication : 1 juillet 2013

Pagination : 235-245

ISSN : 0039-2944

\section{Référence électronique}

Bruno Tribout, « Retz et Mazarin: polémique et politique dans les "Mémoires" de Retz », Studi Francesi [En ligne], 170 (LVII | II) | 2013, mis en ligne le 30 novembre 2015, consulté le 31 janvier 2023. URL: http://journals.openedition.org/studifrancesi/2941 ; DOI : https://doi.org/10.4000/studifrancesi.2941

\section{(c) (i)}

Creative Commons - Attribution - Pas d'Utilisation Commerciale - Pas de Modification 4.0 International - CC BY-NC-ND 4.0

https://creativecommons.org/licenses/by-nc-nd/4.0/ 


\title{
Retz et Mazarin: polémique et politique dans les "Mémoires" de Retz
}

\begin{abstract}
This paper analyses the significance of the image of Mazarin in Retz's Memoirs. Critics have often considered the caricature of the Cardinal as a means for Retz to obliterate the contradictions of his own 'heroic mythology', especially by reinforcing the opposition between his supposed aristocratic ethics and the Machiavellianism attributed to Mazarin. Indeed criticism of Mazarin's hypocrisy, dissimulation and incompetence - an inverted mirror for Princes - enables Retz, by contrast, to give a flattering image of himself in keeping with the antagonistic nature of his defence. Yet the latter cannot conceal the fact that there are significant similarities between Retz and Mazarin, for Retz's criticism of the Cardinal coexists with praise of the author's own skill in political realism. The Memoirs thus rehabilitate not only the notion of honest dissimulation as a means to protect oneself, but also the very Machiavellianism attributed to Mazarin. In combining nostalgia for heroic ideals with political realism (Retz and Mazarin being, in turn, in opposition to and in competition with each other), the comparison between these two enemies proves to be a key element in the missing self-portrait of Retz in his Memoirs, providing insights into his polemical narrative and political ideas.
\end{abstract}

Questo articolo analizza la funzione del ritratto di Mazarin nei Mémoires di Retz. La critica ha spesso considerato la caricatura del ministro come un modo per Retz di eliminare le contraddizioni della propria "mitologia eroica", inasprendo soprattutto l'opposizione tra la sua supposta etica aristocratica e il machiavellismo attribuito a Mazarin. Certo è che l'accusa di ipocrisia, di dissimulazione e di incompetenza del ministro - vero e proprio "specchio del principe" in negativo - permette a Retz di tracciare un'immagine di sé adulatrice, mostrando la natura antitetica della sua apologia. Quest'ultima tuttavia non può dissimulare le profonde similitudini tra Retz e Mazarin: la critica al cardinale coesiste in Retz con una dimostrazione di competenza nell'ambito del pragmatismo politico. I Mémoires riabilitano pertanto sia il concetto della dissimulazione onesta come risposta difensiva, sia lo stesso machiavellismo attribuito a Mazarin. Articolando nostalgia per l'ideale eroico e pragmatismo politico (rispettivamente nell'opposizione e competizione tra Retz e Mazarin), la complessa comparazione tra i due nemici costituisce dunque un elemento chiave del mancante autoritratto di Retz nei suoi Mémoires, permettendo di comprendere meglio la sua argomentazione polemica e le idee politiche.

Retz n'est pas tendre envers Mazarin. En écrivant ses Mémoires, une quinzaine d'années après la mort de ce dernier, l'ancien Frondeur qui n'est jamais rentré en grâce, exilé sur ses terres de Commercy, n'a semble-t-il rien perdu de sa rancœur contre l'ennemi d'autrefois dont il trace un portrait au vitriol: fourbe, insolent, «amuseur en titre d'office» ${ }^{1}$, «ignorantissime» (202) en tout ce qui touche aux institutions

(1) Cardinal de Retz, Mémoires, dans Euvres, éd. Marie-Thérèse Hipp et Michel Pernot, Paris, Gallimard, 1984, p. 827. Toutes les citations des Mémoires renvoient à cette édition, les deux der- niers volumes des Euvres complètes, éditées par Jacques Delon dans la collection «Sources classiques» chez Champion, n'ayant pas paru à ce jour. 
politiques du royaume, véritable bouffon de comédie (197), Mazarin apparâtt en fin de compte comme le grand responsable des maux de la France - Retz contribuant, à travers ce portrait, à la constitution d'une tradition historiographique tenace, comme le montrent les études sur la «réception» du Cardinal' ${ }^{2}$. Si l'on considère l'animosité et souvent la mauvaise foi qui caractérisent l'élaboration de cette légende noire de Mazarin, on pourra certes comprendre les Mémoires de Retz comme l'ultime incarnation du combat d'une vie, où priment naturellement «le souci apologétique» dont Hubert Carrier a souligné qu'il était fréquemment au fondement du genre ${ }^{3}$, voire la portée polémique, étudiée récemment par Myriam Tsimbidy, pour qui le texte, véritable «mise à mort» de Mazarin, se présente comme «une immense machinerie qui sert à démontrer l'insignifiance d'un ministre qui n'a réussi que par hasard $\gg^{4}$. Pourtant, de l'engagement de l'un pour l'exil de l'autre, et vice versa, de la haine publique aux «paix fourrées» (181) qu'ils firent parfois ensemble, les rapports entre Retz et Mazarin sont bien plus complexes qu'il n'y parât à première vue.

On peut penser tout d'abord aux évidents parallélismes entre les deux cardinaux, dont témoigne notamment la biographie de Retz par Simone Bertière et dont Malina Stefanovska a résumé les principaux éléments: chez l'un et l'autre, on note en effet l'origine italienne, des attaches familiales dans l'Église, un engagement réticent dans la carrière ecclésiastique, le goût pour les conquêtes féminines, l'amour des arts et des lettres, enfin et surtout une profonde ambition politique 5 . Toutefois, ces apparentes similitudes biographiques, qu'il convient d'ailleurs de nuancer, Retz ne les met pas en avant dans ses Mémoires, à l'exception sans doute du dernier élément, l'ambition, et ce au point que la Fronde puisse apparaître, écrit Malina Stefanovska, comme «l'histoire de deux frères jumeaux engagés dans une lutte à mort» ${ }^{6}$. Passant du plan biographique au plan symbolique des valeurs du mémorialiste $^{7}$, on peut sans doute reconnaître dans cette lutte à mort - le «Meurs ou tue»

(2) En rappelant que, «en son temps et depuis lors, cet homme a été souvent honni et traîné dans la boue, même par des historiens par ailleurs sérieux» (Mazarin, Paris, Fayard, 1990, p. 445), Pierre GOUBERT souligne en effet que Retz fut un maillon essentiel dans la propagation de cette légende noire de Mazarin: relais entre les pamphlets de la Fronde et Saint-Simon, les propos acerbes de Retz semblent trouver écho jusque chez Michelet, qui parle de Mazarin, ce «fourbe Italien», comme du «roi des fripons», voire chez Adolphe Chéruel, premier historien moderne de la Fronde et admirateur du Cardinal, qui en critique néanmoins «les ruses, les artifices mesquins, les fourberies, l'espionnage» (cités par Pierre Goubert, op. cit., p. 501). Cette durable image négative de Mazarin semble donner raison à Sainte-Beuve, pour qui le malheur du Cardinal fut de n'avoir laissé à sa mort «qu'un seul ennemi avec qui il ne se soit pas réconcilié et à qui il n'a jamais pardonné, le cardinal de Retz», dont les «immortels Mémoires» dressent de Mazarin un «portrait si gai, si vif, si amusant, si flétrissant que les meilleures raisons historiques ont peine à tenir contre l'impression qui en résulte et qu'elles ne parviendront jamais à en triompher» (cité par Georges Dethan, Mazarin, un homme de paix à l'âge baroque (1602-1661), Paris, Imprimerie Nationale, 1981, p. 292).

(3) H. CARRIER, «Pourquoi écrit-on des Mémoires au XVII ${ }^{\mathrm{e}}$ siècle? L'exemple des mémorialistes de la Fronde», dans Cardinal de Retz, Mémoires. Anthologie critique, éd. Pierre Ronzeaud, Paris, Klincksieck, 2005, p. 74.

(4) M. Tsimbidy, Le Cardinal de Retz polémiste, Saint-Étienne, Publications de l'Université de Saint-Étienne, 2005, p. 446.

(5) M. StefanovsKa, La Politique du cardinal de Retz: passions et factions, Rennes, Presses Universitaires de Rennes, 2008, pp. 89-90. Voir également S. Bertière, La Vie du cardinal de Retz, Paris, Éditions de Fallois, 1990. De cette dernière, on signalera la communication concernant «Mazarin vu par Retz dans ses Mémoires», lors du colloque «Nouveaux regards sur les Mémoires du cardinal de Retz», organisé par Jean Garapon, à Nantes, les 17 et 18 janvier 2008, et dont la publication est annoncée chez Cécile-Defaut.

(6) M. Stefanovska, La Politique du cardinal de Retz, cit., p. 89.

(7) Sur les Mémoires comme miroirs des valeurs et des conflits de valeurs nobiliaires, on verra notamment Les Valeurs chez les mémorialistes français du XVII siècle avant la Fronde, éd. Noémi Hepp et Jacques Hennequin, Paris, Klincksieck, 1979 , pp. 153-285, ainsi que la stimulante discussion de la caractérisation des Mémoires comme genre aristocratique dans C. JOUHAUD, D. RIBARD et N. SCHAPIRA, Histoire, Littérature, Témoignage. Écrire les malheurs du temps, Paris, Gallimard, 2009, pp. 23-88. 
de Don Diègue - l'une des caractéristiques de l'éthique héroïque, dont André Bertière a étudié l'importance chez Retz ${ }^{8}$. C'est précisément dans cette perspective que Georges Dethan commentait la complexité de la gémellité, plus symbolique que réelle, entre les deux cardinaux puisque, si l'histoire a tranché en désignant le mémorialiste comme le grand perdant de la Fronde, tout montre au contraire, dans les Mémoires, que Mazarin, véritable anti-héros, n'était pas un ennemi à la hauteur de Retz: soulignant le caractère de ce fait paradoxal de la plaidoirie du mémorialiste? Georges Dethan, sensible aux accents cornéliens de cette éthique héroïque, se demande finalement si Retz n'aurait pas préféré l'échafaud d'un Cinq-Mars comme ultime sanction d'un destin hors pair ${ }^{10}$.

«C'est du Corneille», certes, affirme également Simone Bertière, mais elle ajoute aussitôt: «raconté par un homme d'esprit» ${ }^{11}$, car la lutte à mort entre ces frères ennemis prend, dans les Mémoires, la forme d'un récit enlevé où la grandiloquence du discours sur la magnanimité est souvent contrebalancée par l'humour, par l'introspection, par la spéculation de cet «historien du possible» ${ }^{12}$, par une réflexion pragmatique sur l'action politique, autant d'éléments qui rendent possible une mise à distance des valeurs héroïques. En effet, si ces dernières permettent souvent à Retz de justifier son engagement dans la Fronde, André Bertière a montré que le mémorialiste ne pouvait pas ne pas être conscient des contradictions qui minent cette «mythologie hérö̈que» et expliquent sa faillite non seulement comme principe d'action mais encore comme «principe de cohérence du protagoniste» dans les Mémoires ${ }^{13}$. C'est sur ce terrain, nous semble-t-il, que la gémellité contrariée entre Retz et Mazarin est riche d'enseignements. Certes, la critique a souvent considéré la fonction en quelque sorte négative du portrait de Mazarin, notamment en soulignant qu'il permettait au mémorialiste de gommer les contradictions de cette «mythologie héroïque» en durcissant l'opposition entre machiavélisme d'une part (un ministre pragmatique recherchant l'efficacité politique, quels qu'en soient les moyens) et héroïsme de l'autre (le mémorialiste en quête de gloire, attaché à l'honneur et au bien commun); mais on s'est assez rarement penché sur le contenu positif qui ressort du parallèle entre ces frères ennemis, alors même qu'une telle approche a été adoptée pour étudier les rapports entre Retz et d'autres figures historiques qui lui furent parfois opposées, notamment Condé, Anne d'Autriche ou Gaston d'Orléans ${ }^{14}$.

Dans cette perspective, même si l'image négative du ministre est celle qui, incontestablement, domine les Mémoires, Mazarin se voit parfois attribuer certaines compétences politiques et une telle réhabilitation est paradoxale à plus d'un titre. D'une part, elle semble opposée à la portée polémique du texte et contredire un des fondements les plus efficaces de celle-ci, à savoir la fixité et la cohérence des person-

(8) A. BerTiÈre, Le Cardinal de Retz mémorialiste, Paris, Klincksieck, 1977, pp. 323-349.

(9) Dans cette perspective, Georges Dethan relève que, en quelque sorte par la force des choses, «dans l'exposé des faits, Retz s'est montré souvent moins injuste que dans ses jugements péremptoires et méprisants» (G. Dethan, Mazarin, cit., p. 293).

(10) Ibid., p. 299.

(11) S. BerTIĖRE, La Vie du cardinal de Retz, cit., p. 159. Dans ce passage, l'historienne commente la manière dont Retz évoque le «mouvement intérieur», véritable débat cornélien entre ce qu'il doit à la reine et ce qu'il doit à son «honneur», qui conduisit finalement le coadjuteur à se déclarer contre Mazarin au lendemain de l'arrestation de Broussel (RETz, Mémoires, cit., p. 212 et p. 227).
(12) Pour reprendre l'expression de J.T. LETTS, Le Cardinal de Retz, historien et moraliste du possible, Paris, Nizet, 1966.

(13) A. BERTIĖRE, Le Cardinal de Retz mémorialiste, cit., pp. 350-392.

(14) Sur ces deux derniers personnages, voir P. DANDREY, Commentaire comparé de deux portraits tirés des "Mémoires" du cardinal de Retz, «L'Information littéraire», 40/1 (1988), pp. 26-33. Du point de vue des figures du passé, on peut songer également au rôle joué par César dans les Mémoires (voir A. BeRTIÈRE, Le Cardinal de Retz mémorialiste, cit., p. 363 et l'article de Mrriam Tsimbidy sur Retz et l'image de César, à paraître dans Les Représentations de Jules César à l'époque moderne, Québec, Presses de l'Université Laval, sous presse). 
nages retziens ${ }^{15}$. D'autre part, la simulation et la dissimulation, le machiavélisme, la pratique du «coup d'État» paraissent directement opposés au respect de la bonne foi et de la justice, à l'autorité «tempérée» (193) du pouvoir dans la tradition libérale de la monarchie dont Retz se fait le défenseur $(193 ; 199)$, enfin et surtout à l'éthique de la gloire à laquelle Retz semble vouloir, jusqu'au bout, souscrire et correspondre. Or, condamnées en apparence, ces pratiques du ministre sont pourtant, on le sait, celleslà mêmes que le coadjuteur utilise pour parvenir à ses fins, en le reconnaissant ou non, comme en témoigne l'image d'un Retz machiavélien qui s'est fréquemment imposée à la critique ${ }^{16}$. Si l'on prend en compte cet autre visage de Retz, tout se passe alors comme si la réhabilitation paradoxale de la figure politique de Mazarin ne s'opposait pas au plaidoyer pro domo, mais au contraire permettait au mémorialiste de montrer sa supériorité par rapport à Mazarin jusque sur le terrain du machiavélisme et, partant, de légitimer certaines pratiques politiques faisant partie de son arsenal stratégique pendant la Fronde. C'est en ce sens que le parallélisme latent entre ces frères ennemis nous semble contribuer à l'«autoportrait manquant» du mémorialiste ${ }^{17}$ et permet de se pencher à nouveau sur la question, soulevée par André Bertière, du rôle des valeurs héroïques de Retz comme «principe de cohérence du protagoniste» dans les Mémoires, notamment en restituant l'expression de ces valeurs à leur contexte polémique.

Revenons, dans un premier temps, sur l'aspect le plus visible du parallèle: la manière dont la critique de Mazarin, qui reprend souvent, comme l'a montré Myriam Tsimbidy, les arguments développés dans les pamphlets de la Fronde, est mise au service du plaidoyer de Retz. L'un des traits les plus caractéristiques de la rhétorique judiciaire tournée contre Mazarin concerne le portrait moral qui permet au mémorialiste de reprendre, après les Frondeurs, certains lieux communs de la critique des favoris et principaux ministres ayant cours avant la Fronde et avant Mazarin, tout en personnalisant au maximum l'attaque contre le Cardinal au «vilain cœur» (198). En ce sens, la disqualification de l'adversaire passe d'abord par la réduction de Mazarin à un caractère. Reprenant les lieux communs des caractères des Nations et l'antiitalianisme des pamphlets frondeurs, Retz souligne malicieusement tous les travers que le Cardinal partagerait avec ses compatriotes: à la fois orgueilleux, fanfaron et fourbe, Mazarin apparaît de plus comme un lâche. Veulerie et fierté sont d'ailleurs l'envers et l'endroit de la même médaille, car c'est parce qu' «il avait souvent peur» (198) que Mazarin était vaniteux. En ce sens, le mémorialiste ne manque pas une occasion de souligner la contradiction chez le ministre entre l'être et le paraître. Il se fait, par exemple, un plaisir de rapporter le bon mot de Condé, peu suspect de lâcheté, qui «trouvant un jour [Mazarin] au cercle, et voyant qu'il faisait le fier plus qu'à l'ordinaire, lui dit en sortant du cabinet de la reine, d'un ton assez haut: "Adieu, Mars!” (444). L'ironie de Condé fonctionne comme celle de Retz. Elle consiste dans un mécanisme de dissociation entre la vérité des qualités morales de Mazarin, d'une

(15) Voir notamment P. SABBAH, La Galerie des Illustres dans les Mémoires du Cardinal de Retz, Saint-Étienne, Publications de l'Université de Saint-Étienne, 2000, pp. 73-82. De tous les acteurs historiques dépeints par Retz, il semble que Mazarin soit d'ailleurs le plus rigide, et le moins sujet aux contradictions étudiées par Pierre Sabbah (Ibid., pp. 111-114). Sur le portait dans les Mémoires, voir également E. Lesne-Jafrro, La Poétique des Mémoires, Paris, Champion, 1996, pp. 115-160.

(16) On notera, par exemple, la manière symbolique dont, en introduisant son enquête sur les Mémoires d'Etat, P. Nora évoque, parmi les grands mémorialistes ayant pu influencer De Gaulle, «Retz pour la vision machiavélienne» («Les Mémoires d'État de Commynes à de Gaulle», dans Les Lieux de mémoire, éd. Pierre Nora, Paris, Gallimard, 1986, vol. II: La Nation, t. 2, p. 355). Voir également A. BerTIĖRE, Le Cardinal de Retz mémorialiste, cit., pp. 355-363.

(17) Sur les raisons de cette absence, voir RETZ, Mémoires, cit., p. 292, E. Lesne-Jaffro, La Poétique des Mémoires, cit., p. 151 ainsi qu'I. TRIVISANI-MOREAU, L'autoportrait manquant dans les "Mémoires", «Littératures classiques», 57/2 (2005), pp. 69-84. 
part, et, de l'autre, les apparences de vertu qu'il affiche pour se rendre digne de sa fonction. Or cette argumentation ad hominem, Retz en pousse l'utilisation polémique plus loin dans les Mémoires qu'il ne le fit dans les pamphlets de la Fronde, comme l'a montré Myriam Tsimbidy. Au dire de Retz, en effet, Mazarin «porta le filoutage dans le ministère, ce qui n'est jamais arrivé qu'à lui; et ce filoutage faisait que le ministère, même heureux et absolu, ne lui seyait pas bien, et que le mépris s'y glissa, qui est la maladie la plus dangereuse d'un État» (198). Véritable «coup textuel» ${ }^{18}$, la dissociation est telle entre les qualités requises pour un ministre d'État et les qualités effectives de Mazarin que non seulement sa fonction, mais le corps de l'État tout entier en pâtissent; le résultat de cette dissociation s'exprime alors à travers la notion à la fois morale et esthétique de ridicule, dont les Mémoires couvrent Mazarin.

En ce sens, le texte fournit un véritable répertoire des bévues politiques du Cardinal, sorte de miroir des Princes en négatif, qui reprend en les renversant les lieux de l'éloge, dont se servait notamment la propagande même de Mazarin ${ }^{19}$. On voit par exemple en quelle estime Mazarin tient la vertu de tempérance, essentielle pour l'exercice du pouvoir. Non seulement, selon Retz, il ne met aucun frein à sa domination, mais il semble se plaire à galvauder la modération, la promettant toujours mais ne l'accordant jamais. Au moment de la journée des barricades, on apprend ainsi comment Retz fut, selon ses termes, «joué» parce que les «mouvements de modération et de douceur» (215) promis par la cour et censés accompagner la victoire de Lens ne furent qu'un leurre de Mazarin pour amuser le Parlement. Si le ministre n'a pas l'humeur aigre et bilieuse de la reine, cela n'empêche pas que tel libelle soit parfois décrit comme «une saillie de la fureur de M. le cardinal Mazarin» (581); loin d'être «doux comme un agneau» (185), comme le prétend la reine, Mazarin cache, sous une feinte douceur, une animosité rentrée et un acharnement immodéré à poursuivre ses buts. En outre, et plus encore que l'absence de tempérance, c'est le manque de prudence qui fait de Mazarin un piètre politique. Celui dont «il faudrait, au dire de Retz, des volumes pour vous raconter toutes [1] es fautes» (198), ne cesse en effet d'agir à contretemps, de prendre des risques inutiles, jusqu'à avoir eu, lors des troubles de Guyenne, par exemple, l'«imprudence [d'] avoir hasardé tout le royaume» en une seule bataille (569). À côté d'un manque de prévoyance et d'intelligence politique, l'imprudence de Mazarin tient plus fondamentalement, selon Retz, à une espèce d'amateurisme dont témoigne sa profonde ignorance à la fois des mœurs politiques françaises et du fonctionnement des institutions du royaume - argument qui, comme l'a montré Myriam Tsimbidy, constitue un «des leitmotive propres aux pamphlets retziens» transcrits dans les Mémoires ${ }^{20}$. Cette ignorance politique du ministre inspire à Retz les termes les plus dépréciatifs: en méjugeant l'importance des conflits de juridiction entre la cour des aides et le Parlement, Mazarin s'attire par exemple l'étiquette d'«ignorantissime en toutes ces matières» (202). Quant à l'incapacité du Cardinal à être juste, elle dérive de son ignorance et de son imprudence, mais est encore aggravée par la nature même de son caractère: en effet, se souvenant

(18) M. Tsimbidy, Le Cardinal de Retz polémiste, cit., p. 450.

(19) Sur les vertus, voir ARISTOTE, Rhétorique, 1366b, THOMAs D’Aquin, Somme théologique, 2a 2ae, Question 123, Paris, Desclée, 1926, pp. 50-51 et, pour une synthèse, J.-M. AUBERT, art. «Vertus», Dictionnaire de spiritualité ascétique et mystique, Paris, Beauchesne, 1932-1995, t. 16, col. 485-497. Sur l'utilisation de ces lieux de l'éloge dans les genres épidictiques à l'âge classique, voir $\mathrm{P}$. ZoBerman, Les Panégyriques du roi, Paris, Presses de l'Université Paris-Sorbonne, 1991, pp. 30-33 et, pour leur rôle dans la propagande de Mazarin, Y. LoskoutofF, «Les vertus cardinales», Rome des Césars, Rome des Papes: la propagande du cardinal Mazarin, Paris, Champion, 2007, p. 24 et pp. 27197 et M. LAURAIN-PorTEMER, Etudes mazarines II: Une tête à gouverner quatre empires, Paris, chez l'auteur; diffusion: Nogent-le-Roi, Jacques Laget, 1997, pp. 759-1002.

(20) M. Tsimbidy, Le Cardinal de Retz polémiste, cit., pp. 329-330. 
davantage des injures que des bienfaits, Mazarin est présenté comme «ingrat au dernier point» (211). On comprend donc, ultimement, ce qui mine l'exercice du pouvoir par le ministre: en le rapportant à sa propre personne plus qu'au bien du royaume, il n'est pas seulement ridicule comme on l'a vu plus haut, mais encore et surtout «despotique». Aussi, pour achever de noircir son ennemi, le mémorialiste n'a-t-il de cesse de recycler la rhétorique anti-tyrannique des pamphlets frondeurs corrélant les maux présents de la monarchie et la montée en puissance d'un ministre «favori» ${ }^{21}$. Poursuivant «l'établissement de l'autorité purement et absolument despotique» (199), entrepris par «les ministres du dernier siècle» (490) et qui déboucha avec Richelieu sur «la plus dangereuse tyrannie» (194), mais sans avoir pourtant l'intelligence de son prédécesseur, Mazarin apparaît finalement, selon une formule prêtée à Gaston d'Orléans, comme «un ministre incapable et abhorré du genre humain» (774).

Or cet impressionnant répertoire des «fautes» (198) d'un ministre despotique, Retz s'en sert non pas pour défendre réellement un certain art de gouverner, mais plutôt comme d'un faire-valoir lui permettant de renvoyer une image de lui-même flatteuse. L'autoportrait manquant de Retz pourrait donc se donner ici à lire en filigrane, obéissant aux mêmes règles que «le parallèle» (196) entre les cardinaux-ministres au sujet desquels Retz écrivait que l'un était «d'un caractère tout contraire» (197) à l'autre. En ce sens, on peut penser que le ridicule dont Retz couvre Mazarin «permet, en négatif, comme l'écrit Jean Garapon, de reconstituer en quelque sorte un portrait idéal du héros-narrateur ${ }^{22}$, et que les vertus cardinales et l'intelligence politique qui font défaut chez Mazarin dessinent en creux le portrait du grand politique que serait Retz. Ainsi la couardise du ministre relève-t-elle les couleurs du courage dont fait preuve le coadjuteur, qu'il s'agisse d'affronter tous les diables de l'anecdote des augustins déchaussés (161-64) ou de plus réels dangers, comme cette barricade où Retz faillit être tué par «un garçon d'apothicaire» (222) ou bien encore cette troupe de «séditieux» qui réclament sa mort dans la cour de l'hôtel de Gaston d'Orléans (794). L'intrépidité de Gondi n'est pourtant pas suivie de vaine gloire. Face à l'arrogante fierté du Cardinal, Retz tâche en effet de se tenir dans les bornes d'une honnête modestie. S'il ne ménage pas ses efforts pour faire respecter les prérogatives de sa charge, même, avoue-t-il, avec un peu trop de zèle à ses débuts, il sait également renoncer de bonne grâce à des prétentions légitimes, lorsqu'elles pourraient paraître excessives. Retz explique ainsi que les cardinaux-ministres, «qui avaient confondu le ministériat dans la pourpre, avaient attaché à celle-ci de certaines hauteurs qui ne conviennent à l'autre que quand elles sont jointes ensemble. Il eût été difficile de les séparer en ma personne, au poste où j'étais à Paris. Je le fis de moi-même» (809). On ne peut que souligner le caractère rhétorique d'une telle démonstration de modestie; elle suppose, bien entendu, que l'on accepte l'élément contestable sur lequel repose ce pseudo-syllogisme: à savoir que le pouvoir de Retz dans Paris égale celui des ministres... Il reste qu'on observe, à travers ce raisonnement, la structure antithétique de la plaidoirie retzienne, dont témoigne encore le rôle que Retz fait jouer à l'imprudence de Mazarin, ou à ses impostures. En effet, Retz montre que le ministre, par ses «artifices» et «piperies», en impose à la reine en même temps qu'au «public». À l'inverse, Retz prend toujours soin de souligner, même au cœur de la guerre civile, qu'il respecte profondément l'autorité royale incarnée dans la régente. Aux premières heures des troubles parisiens, il écrit ainsi: «je crus que je devais sacrifier à la reconnaissance et

(21) Sur la question du «ministériat», voir H. CARRIER, Le Labyrinthe de l'État. Essai sur le débat politique en France au temps de la Fronde, Paris, Champion, 2004, pp. 263-269.
(22) J. GARAPON, «Le ridicule, une notion-clé des Mémoires du cardinal de Retz», dans Mémoires des XVII et XVIII siècles: nouvelles tendances de la recherche, Tours, Université de Tours, 2003, p. 13. 
mes ressentiments et même les apparences de ma gloire» (212); plus loin, il propose encore à la reine de tâcher de rétablir l'autorité royale en se sacrifiant lui-même (650). C'est que la reconnaissance et la fidélité de Retz ont pour corollaires le loyalisme monarchique et le sentiment de la justice ${ }^{23}$. En ce sens, tandis que Mazarin apparaît comme le despote qui renverse l'État, Retz se pose en gardien du «bien public» pour l'intérêt commun du roi et de ses sujets.

Ne doit-on pas, cependant, considérer ce parallèle, biaisé en raison de sa portée polémique, comme une de ces «ruses et [...] stratagèmes que Retz [...] mi[t] en œuvre pour se montrer à la postérité tout autre qu'il ne fut en réalité pour ses contemporains $\gg^{24}$, comme l'écrivait, à la fin du XIX ${ }^{\mathrm{e}}$ siècle, Régis de Chantelauze, rappelant ainsi la tension constante entre sincérité et création dans les Mémoires, tension qui, on le sait, devait particulièrement retenir l'attention des critiques? ${ }^{25}$ Dans ce domaine, pourtant, ce qui frappe à propos du «stratagème» textuel que constitue en un sens le parallèle constamment suggéré entre Retz et Mazarin, ce n'est pas tant son évidente mauvaise foi à l'endroit du ministre que la grossièreté de ses ficelles, au point que l'on peut se demander si cette élaboration d'un autoportrait en creux, inversement symétrique par rapport à celui de Mazarin, n'a pas également pour fonction, non plus de masquer, mais au contraire de révéler les similitudes qui ne peuvent manquer d'apparaître si l'on considère de plus près la conduite politique de ces frères ennemis. Le premier élément frappant en ce sens a trait à l'usage de la dissimulation: tout se passe comme si Retz, autant que Mazarin, avait adopté ce «grand principe» sur lequel s'ouvre le satirique Bréviaire des politiciens: «Simule et dissimule» ${ }^{26}$. Certes, le mémorialiste a beau jeu de décrire Mazarin, ici à travers Monsieur, comme «un menteur fieffé que personne ne croit» (827), de voir partout «quelque méchante rouse du Mazarin» (317) et de stigmatiser en lui «l'homme du monde qui reçût toujours le plus agréablement, en apparence, ce qu'il ne voulait pas en effet» (661); mais que dira-t-on de l'attitude souvent retorse de Retz? Pris entre Gaston d'Orléans et le Parlement, entre Monsieur et Monsieur le Prince, entre la reine et Condé, orchestrant d'improbables alliances et rêvant d'un «tiers parti» (748), Retz aura finalement trompé un peu tout le monde tour à tour, au moins autant que son pire ennemi. Pourtant, en dépit de ces «mensonges» soulignés par toute une tradition critique ${ }^{27}$, le mémorialiste ne cherche pas, dans la plupart des cas, à maquiller son recours à la dissimulation. En ce sens, on retrouve dans les Mémoires une réhabilitation de la dissimulation proche de celle que l'on observe dans la propagande même de Mazarin. Comme l'a montré Yvan Loskoutoff $^{28}$, cette dernière reflète à sa manière la «querelle de la prudence» qui oppose alors, schématiquement, une haute prudence dérivée d'Aristote, aimantée par l'idée du bien, à une prudence faite de finesse et d'intrigues, subordonnée à l'intérêt et tenue pour machiavélique ${ }^{29}$. Or la ligne de démarcation entre ces deux

(23) Sur l'importance, dans l'argumentation des Frondeurs, du couple justice-prudence au détriment du couple justice-force, et sur le bouleversement consécutif non seulement de la hiérarchie des vertus mais de leur légitimité même, voir Y. Loskoutoff, Rome des Césars, Rome des Papes, cit., pp. $129-133$.

(24) R. DE Chantelauze, «Avertissement», Euvres du cardinal de Retz, Paris, Hachette, 1887, t. IX, p. IV.

(25) À ce sujet, voir les remarques de S. BERTIÈRE dans son essai bibliographique: Le Cardinal de Retz, Rome, Memini, 2000, p. 153.

(26) Bréviaire des politiciens [Breviarium politico- rum secundum rubricas Mazarinicas], trad. Florence Dupont, Langres, Café-Clima, 1984, p. 17.

(27) Hubert Carrier en a rappelé les grandes étapes et les principes critiques dans son article: Sincérité et création littéraire dans les Mémoires du cardinal de Retz, «XVII siècle», 94-95 (1971), pp. 39-74.

(28) Y. Loskoutoff, Rome des Césars, Rome des Papes, cit., pp. 92-135.

(29) Sur cette opposition, on verra E. GARVER, Machiavelli and the History of Prudence, Madison, University of Wisconsin Press, 1987, C. LaZZERI, «Prudence, éthique et politique de Thomas d'Aquin à Machiavel» dans De la prudence des 
prudences n'est pas rigide et nombre d'arguments furent employés, dans les pamphlets de la Fronde, pour permettre à la première d'emprunter à la seconde; la «prudence mixte» qui en résulte, inspirée par Lipse ${ }^{30}$, joue sur les différents degrés qui séparent dissimulation et simulation ${ }^{31}$ pour justifier, notamment, l'usage d'une «honnête dissimulation». C'est derrière celle-ci que Retz semble s'abriter dans un premier temps.

Dans l'optique d'une morale héroïque, il importait en effet au mémorialiste de justifier le recours intermittent à la dissimulation, qui heurtait de front la notion aristocratique de conflit ouvert. Deux arguments sont mis à contribution en ce sens. Il s'agit parfois de taxer l'ennemi d'avoir eu l'initiative de la trahison. Ainsi, c'est la reine, c'est Condé qui ont d'abord manqué à Retz, l'obligeant à prendre des mesures pour se tirer lui-même d'affaire. Mais le mémorialiste se garde bien d'abuser de ce type d'argument qui l'apparente trop évidemment à ses ennemis. Il préfère, de loin, recourir à l'argument du sacrifice: c'est pour le bien du «parti» qu'il se voit parfois contraint, à regret, de recourir à la ruse et à la dissimulation, en dépit de ce qu'une telle attitude peut coûter à ses principes. Exemplaire en ce sens est l'argumentation visant à justifier à la fois la nécessité de faire alliance avec l'Espagne et celle de dissimuler pour faire agréer au Parlement l'alliance en question. En guise d'entrée en matière, Retz prend soin de se dédouaner d'être à l'origine des négociations avec l'ennemi espagnol: en effet, c'est Mazarin qui lui ouvre la voie, envoyant un émissaire «en Flandres pour commencer quelque négociation avec les Espagnols» (304). D'autre part, ce n'est pas l'intérêt particulier, mais le bien commun de la lutte contre Mazarin qui impose aux Frondeurs un traité avec l'ennemi, «que nous étions obligés de recevoir comme un remède à nos maux» (310). Comme il juge plus prudent que ce remède soit d'abord purifié en passant par «l'alambic du Parlement» (310), Retz met en place une stratégie de dissimulation en deux temps, destinée à ôter tout soupçon à la «compagnie»: il dupe à la fois les Frondeurs et le Parlement, d'une part en faisant parvenir aux généraux de la Fronde une missive espagnole de commande et, d'autre part, en dictant à l'envoyé espagnol «tout ce qu'il devait dire au Parlement» (310). On voit donc que, sur un sujet pourtant sensible, impliquant un crime de lèse-majesté $e^{32}$, Retz ne ménage pas sa peine pour tromper, et tromper y compris son propre camp, tout en sauvant les apparences. Qui plus est, le mémorialiste prend soin de souligner les succès de l'artifice. En effet, si «la proposition [d'écouter l'envoyé] fut reçue d'abord comme une hérésie» (310), elle débouche finalement sur «ce que nous n'eussions pas seulement osé imaginer, qui est l'union de l'Espagne et du Parlement pour notre défense» (354). S'abritant tant bien que mal derrière l'idée d'honnête dissimulation justifiée par la nécessité du bien commun, le double stratagème de Retz débouche en réalité sur une démonstration de compétence établissant sa maîtrise machiavélienne des techniques du secret.

En ce sens, la dissimulation et la ruse nous confrontent à un paradoxe: blâmées chez Mazarin, elles servent d'argument au mémorialiste pour faire l'éloge de sa propre habilité politique. Dans l'optique polémique, cette ambivalence ne semble

anciens comparée à celle des modernes: sémantique d'un concept, déplacement des problématiques, éd. André Tosel, Besançon, Annales Littéraires de l'Université de Besançon, 1995, pp. 79-128 et S. Gouverneur, Prudence et subversion libertines, $\mathrm{Pa}$ ris, Champion, 2005, pp. 131-141.

(30) Juste Lipse, Les Politiques. Livre IV, trad. Charles Le Ber [1597], éd. Jacqueline Lagrée, Caen, Presses Universitaires de Caen, 1994, pp. 79-80.

(31) Sur cette question, voir notamment J.-P. CA-
VAILLÉ, Dis/simulations. Jules-César Vanini, François La Mothe Le Vayer, Gabriel Naudé, Louis Machon et Torquato Accetto. Religion, morale et politique au XVII siècle, Paris, Champion, 2002, pp. 11-31.

(32) Sur la question de la trahison, voir D.A. WATTS, «La notion de patrie chez les mémorialistes d'avant la Fronde», dans Les Valeurs chez les mémorialistes français, cit., pp. 195-209 ainsi que S. BERTIĖRE, Retz face à la guerre franco-espagnole, «Littératures classiques», 57/2 (2005), pp. 41-53. 
concevable qu'autant que Retz cherche à montrer que la duplicité procède, chez Mazarin, d'un esprit de fourberie mesquine et égoïste, tandis qu'elle est, chez le Frondeur, l'indice d'une intelligence politique hors pair, mise au profit du public. Or il est clair que cet argument polémique n'est pas vraiment propre à emporter l'adhésion de la destinataire des Mémoires. Et Retz le sait. Certes, il multiplie les déclarations de dévouement sacrificiel au bien commun, notamment en refusant obstinément les «accommodements» particuliers lors des négociations entre les Frondeurs et la cour, mais il ne peut cacher que ses vrais mobiles, dictés purement par l'intérêt, sont la conquête du «chapeau», la lutte pour l'archevêché, sinon peut-être davantage. Or non seulement Retz ne peut occulter qu'il s'attache à son intérêt autant que pouvait le faire son ennemi déclaré, mais il doit encore reconnaître qu'il recourt aux mêmes expédients que Mazarin pour parvenir à ses fins, sans toujours s'inquiéter de la légitimité morale des moyens mis en œuvre. Juxtaposée à l'éthique de la gloire qui justifie parfois les grands crimes en vertu de la grandeur du dessein, on trouve donc dans les Mémoires une réhabilitation non plus de la «prudence mixte» des politiques, mais du machiavélisme même que Retz attribue à son ennemi. Car l'ancien Frondeur montre qu'il lui fallait utiliser les armes appropriées pour obtenir l'éviction du ministre et que celles-ci ne pouvaient être que d'une seule espèce: «l'unique moyen pour y parvenir, dit Retz à travers Gaston d'Orléans, était de bien faire la guerre, et [...], pour la bien faire, il la fallait faire sans scrupule» (784): il convient donc «de lever des hommes et de l'argent, de se rendre maître des bureaux, de se saisir des deniers du Roi» (784), c'est-à-dire qu'il fallait, en somme, «faire comme lui» (784). Le mot est donc lâché: pour parvenir à ses fins et contrer efficacement Mazarin, son alter ego, Retz imitera le ministre sur le terrain du pragmatisme machiavélien. Le mémorialiste a beau se méfier, dit il, de «l'esprit d'un Italien politique par livre» (191), ce sont sans doute les mêmes ouvrages qui inspirèrent la plupart de ses propres maximes. Ce parallélisme entre les deux cardinaux, constamment suggéré en sous-main, un dernier exemple permettra de l'illustrer. Retz souligne que Mazarin aimait agir en déléguant à ses «créatures» qu'il sacrifiait ensuite volontiers pour se protéger, comme lorsque, exilé et cherchant à se faire rappeler, il écrit à la reine de ne «point balancer à éloigner les sous-ministres» (686). Or cette maxime est brillamment appliquée pendant la Fronde par le mémorialiste lui-même, qui, afin de ne pas sacrifier la dignité de sa charge ni s'avancer plus qu'il ne voudrait, utilise Beaufort pour contrôler le peuple de Paris: «j'avais besoin, dit-il, d'un homme que je pusse, dans les conjonctures, mettre devant moi [...]. Il me fallait un fantôme, mais il ne me fallait qu'un fantôme; et par bonheur pour moi, il se trouva que ce fantôme fut petit-fils d'Henri le Grand» (293). Une fois encore, la désinvolture et l'humour des propos de Retz ne sauraient masquer le soin que le mémorialiste apporte à montrer sa compétence sur le terrain même du machiavélisme.

Au terme de ce parcours, la plaidoirie de Retz, souvent réduite à une diatribe acharnée et univoque contre Mazarin, apparaît plus complexe qu'on ne la présente habituellement. Derrière l'opposition de façade entre Retz et Mazarin, il existe un secret parallélisme, fondé paradoxalement sur ce que le mémorialiste feint souvent de blâmer le plus ouvertement chez son ennemi. Pour rendre compte d'un tel paradoxe, on voudrait, pour conclure, formuler deux hypothèses découlant des observations précédentes. La première tient à la nature complexe du plaidoyer de Retz dans les Mémoires. Contrairement à ce que l'on obverse souvent dans les mazarinades, il semble que, dans les Mémoires, sous l'effet de la distance par rapport au passé frondeur et en vertu de la souplesse du genre, Retz juxtapose volontiers des lignes d'argumentation qui apparaissent certes contradictoires sur le plan des valeurs, mais dont la justification repose sur l'efficacité du point de vue polémique ou apologétique. Peuvent ainsi cohabiter idéal héroïque et pragmatisme machiavé- 
lien, non par un tour qui tiendrait d'un certain «impressionnisme princier» pour reprendre le mot de Jean Garapon au sujet de la Grande Mademoiselle ${ }^{33}$, mais parce que tous deux servent tour à tour à lutter contre Mazarin et à montrer la supériorité de Retz sur ce dernier. On objectera que le mémorialiste a beau chercher à se montrer supérieur à cet anti-héros en bien comme en mal qu'est Mazarin, il n'en reste pas moins que Retz ressort perdant de la lutte, tant du point de vue de son action politique proprement dite que des justifications de cette action. Au-delà de l'instrumentalisation polémique de l'héroïsme comme du pragmatisme, il y aurait une irréductible différence de nature entre ces postulations contradictoires, qui résumerait l'échec de Retz. Mais la prise de conscience de ces contradictions et de cet échec ne nous semble pas être le produit des Mémoires à la manière d'une «révélation» qui serait «refusée» par Retz, comme l'écrit André Bertière, notamment en raison de l'attachement du mémorialiste aux idéaux aristocratiques ${ }^{34}$. Présente en amont des Mémoires, nourrie par une réflexion constante sur l'action et influencée par la «démolition du héros» dans l'air du temps ${ }^{35}$, cette conscience aiguë, chez le mémorialiste, des limites de l'héroïsme semble expliquer - et c'est là notre seconde hypothèse, dérivant de la première - que l'hérö̈sme s'exprime d'emblée, chez Retz, sur le mode de la nostalgie et cohabite avec le pragmatisme politique, voire implique ce dernier. Pour se limiter à quelques exemples antérieurs aux Mémoires, on rappellera ainsi comment, dans La Conjuration du comte Jean-Louis de Fiesque, Verrina, dont Fiesque suit les conseils, fait dériver sa critique de la prudence d'une maxime héroïque selon laquelle «le crime d'usurper une couronne est si illustre qu'il peut passer pour une vertu» ${ }^{36}$. Similairement, dans son «Sermon sur la pénitence», trouvant des excuses à l'ambitieux qui bouleverse un État à la poursuite de «l'honneur qui est quasi la seule fin de la vie civile»» ${ }^{37}$, Retz souligne que cette quête héroïque s'appuie nécessairement sur un art de «savoir tromper délicatement», voire sur «une malice étudiée, savante, ingénieuse et inventive» ${ }^{38}$, au point que, pour lui, le crime de l'hypocrite par ambition «est plus pardonnable devant Dieu» ${ }^{39}$ que celui de l'hypocrite par cupidité ou luxure. Ces réflexions éparses dans l'œuvre tendent à montrer que Retz ne cherche pas nécessairement à «concilier les inconciliables» ${ }^{40}$ mais plutôt qu'il n'y a pas, chez lui, solution de continuité entre nostalgie pour l'idéal héroïque et pragmatisme machiavélien. D'emblée parallèle à la ligne d'argumentation qui s'appuie sur les valeurs héroïques, on trouve ainsi dans les Mémoires une démonstration qui vise à souligner la supériorité politique de Retz en montrant qu'il a compris et maîtrisé la nouvelle donne de la morale politique moderne, cellelà même qui permit à Mazarin de s'élever au pouvoir et de s'y maintenir. En ce sens, en articulant pragmatisme politique, par principe de similitude, et nostalgie héroïque, par principe d'opposition, le parallèle constamment suggéré entre Retz et Mazarin joue un rôle essentiel: la Vie du cardinal de Rais cache une manière de

(33) J. GARAPON, La Culture d'une princesse. Écriture et autoportrait dans l'ouvre de la Grande Mademoiselle (1627-1693), Paris, Champion, 2003, p. 379.

(34) A. Bertière, Le Cardinal de Retz mémorialiste, cit., p. 389.

(35) Sur cette question, voir notamment P. BÉNICHOU, «La démolition du héros», Morales du grand siècle, Paris, Gallimard, 1948, pp. $128-148$ et A.H.T. LÉVI, «La disparition de l'héroïsme: étapes et motifs», dans Héroïsme et création littéraire sous les règnes d'Henri IV et de Louis XIII, éd. Noémi Hepp et Georges Livet, Paris, Klincksieck, 1974, pp. 77 88; pour l'empreinte de cette évolution chez Retz:
R. PINTARD, «La Conjuration de Fiesque ou l'héroïsation d'un factieux», dans Héroïsme et création, cit., pp. 225-232 et A. BERTIÈre, Le Cardinal de Retz mémorialiste, cit., pp. 323-392.

(36) CARDinAl DE RETZ, La Conjuration du comte Jean-Louis de Fiesque dans Euvres, éd. Marie-Thérèse Hipp et Michel Pernot, Paris, Gallimard, 1984, p. 25.

(37) Cardinal de Retz, Euvres complètes, éd. Jacques Delon, Paris, Champion, 2005, t. I, p. 410.

(38) Ibid., p. 402.

(39) Ibid., p. 410.

(40) A. Bertière, Le Cardinal de Retz mémorialiste, cit., p. 362. 
Vies parallèles, le mémorialiste nous invitant, comparant chaque fois le comparable, à voir en lui, à la différence du Cardinal, un grand seigneur dont l'hérö̈sme nostalgique est sublimé par l'échec, mais aussi, comme Mazarin ou davantage encore, un grand politique, capable de rivaliser avec l'ennemi sur son propre terrain.

BRUNO TRIBOUT 